\title{
The Relevance of Biopsy in Tuberculosis Patients Without Human Immunodeficiency Virus Infection
}

\author{
Kyung Min Bae, $\uparrow$ Sung-Chul Lim, $†$ Hyung Ho Kim, Woo Jin Lee, Na Ra Yun, Choon-Mee Kim, and Dong-Min Kim* \\ Departments of Internal Medicine and Pathology, Chosun University College of Medicine, Gwangju, South Korea; \\ Division of Natural Medical Sciences, College of Health Science, Chosun University, Gwangju, South Korea
}

\begin{abstract}
Although chronic granulomatous inflammation (CGI) with concomitant caseous necrosis $(\mathrm{CN})$ is a characteristic histological feature of tuberculosis (TB), few studies have investigated its frequency or various pathologic findings. The medical records of 227 human immunodeficiency virus (HIV) -negative, culture-positive TB patients who underwent biopsy were studied. After the frequency of characteristic pathological findings of TB was determined, a pathologist reanalyzed the pathological findings with particular focus on necrosis and reclassified CGI, CN, or possible CN into possible TB pathologic findings. The initial biopsy interpretation revealed that $63(34.8 \%)$ of 181 patients with pulmonary TB had caseating granulomas, $36(19.9 \%)$ patients had only CGI, and $6(3.3 \%)$ patients had only CN. Among 46 patients with extrapulmonary TB, $16(34.8 \%)$ patients had only caseating granulomas, and $14(30.4 \%)$ patients had only CGI. More patients who underwent percutaneous lung biopsy had CGI or CN $(76.3 \%)$ than patients who underwent transbronchial lung biopsy $(53.6 \%)$. The reanalysis confirmed all $\mathrm{CN}$ cases identified by the first interpretation, and $20(95.2 \%)$ of 21 non-CN cases were reclassified as possible CN. Ten cases (three pulmonary and seven extrapulmonary) were reclassified as possible TB pathologic findings from just necrosis. Caseating granuloma was present in only onethird of TB cases. Even in cases where only necrosis was identified, $\mathrm{CN}$ may be present.
\end{abstract}

\section{INTRODUCTION}

Despite centuries-long efforts to conquer tuberculosis (TB), it remains rampant, with approximately 9.2 million new cases annually and an annual death toll of 1.58 million. ${ }^{1,2}$

In TB-endemic regions, rapid and accurate TB diagnosis is crucial for controlling infection. Substantial delays in diagnosis and treatment can occur, however, because TB bacterial culture requires at least 3-6 weeks. A histological finding of chronic granulomatous inflammation (CGI) concomitant with caseating necrosis $(\mathrm{CN}$; hereafter referred to as caseating granuloma) can be strong evidence of active TB, albeit not pathognomonic. ${ }^{3}$

In contrast to pulmonary $\mathrm{TB}$, in which clinical samples, such as sputum, are relatively easy to obtain, diagnosing extrapulmonary TB is challenging, because acid-fast bacillus (AFB) stain and AFB culture tests are positive in fewer than $10 \%$ and $20 \%$ of suspected cases, respectively. ${ }^{4,5}$ Thus, administering antitubercular agents in the early stages may sometimes be necessary in cases in which histological findings are eventually compatible with TB-suspected clinical findings.

A study by Cutler and others ${ }^{6}$ stated that the concordance rate is high between histological findings compatible with TB and microbiological diagnoses, and there was one report wherein CGI was documented in 53 of 81 patients. ${ }^{7}$ However, most similar studies were based on old data and small sample sizes. To date, no study has used a systematic analysis of the biopsy results and a reanalysis of the initial interpretations in patients having TB to investigate histological findings other than those of caseating granuloma, $\mathrm{CN}$, or CGI.

This study aimed to retrospectively determine the concordance rate between the histological findings characteristic of

* Address correspondence to Dong-Min Kim, Department of Internal Medicine, Chosun University College of Medicine, 588 Seosuk-dong, Dong-gu, Gwangju 501-717, South Korea. E-mail: drongkim@chosun .ac.kr

$\dagger$ These authors contributed equally.
$\mathrm{TB}$, such as caseating granuloma, and actual clinical cases by analyzing the biopsy results of patients with cultureconfirmed TB. Additionally, the clinical relevance of biopsy for diagnosing TB was investigated by identifying the histological findings other than caseating granuloma, $\mathrm{CN}$, or CGI that may be indicative of TB.

\section{METHODS}

The medical records of human immunodeficiency virus (HIV) -negative, culture-positive TB patients who presented at Chosun University Hospital and underwent biopsy from January of 2002 to December of 2011 were studied retrospectively.

A confirmatory diagnosis was defined as the presence of Mycobacterium tuberculosis in the culture test. The first analysis was performed on the interpretation sheets for each patient, which were reported by six pathologists. We classified these pathologic reports to identify TB findings in three major categories: caseating granuloma, CGI, and $\mathrm{CN}$. The second analysis was performed by a pathologist who was aware that the cases were cultured to confirm the findings of the three major categories and then reclassified them as the three major categories and possible $\mathrm{CN}$ (PCN). PCN is defined as those cases that were determined to be non-CN, such as simple necrosis, fibrinoid necrosis, coagulation necrosis, and abscess, in the first interpretation but then considered highly likely to be $\mathrm{CN}$ when evaluated by a pathologist who was aware that the case is culture-confirmed TB. All histological findings indicative of possible $\mathrm{TB}$, including the three categories of caseating granuloma, $\mathrm{CGI}, \mathrm{CN}$, and $\mathrm{PCN}$, were defined as possible TB pathologic findings.

Samples for which the recorded site suspected of TB and the actual anatomical location did not coincide were excluded from the analyses as inadequate samples. This study was conducted with the approval of the institutional review board of Chosun University Hospital (IRB 2013-11-009).

A $P$ value of less than 0.05 was considered statistically significant. Analyses were performed using SPSS 12.0 statistical software (SPSS Inc., Chicago, IL). 


\section{RESULTS}

The subjects' baseline characteristics. Culture-confirmed TB was diagnosed in 972 patients, including 231 patients who underwent perilesional tissue biopsies. Analyses were performed on 227 cases after 4 inadequate samples were excluded because of anatomical mismatch. The mean subject age was 59.4 years (range $=18-91$ years), and the male-tofemale ratio was 1.01:1 $(N=114: 113)$.

There were $79.7 \%(N=181)$ and $20.3 \%(N=46)$ pulmonary and extrapulmonary patients, respectively. The latter consisted of tuberculous pleurisy $(N=22 ; 9.7 \%)$, osseous TB $(N=9 ; 4.0 \%)$, genitourinary TB $(N=6 ; 2.6 \%)$, tuberculous lymphadenitis $(N=5 ; 2.2 \%)$, tuberculous pericarditis $(N=2$; $0.9 \%)$, and gastrointestinal TB $(N=2 ; 0.9 \%)$.

The initial biopsy results identified 80 cases of caseating granuloma $(35.2 \%)$, 49 cases of only CGI $(21.6 \%)$, and 6 cases of only CN $(2.6 \%)$. The remaining 92 cases $(40.5 \%)$ showed other findings. Therefore, of a total of 227 patients, only $135(59.5 \%)$ patients were found to have either CGI or CN alone or both together. Reanalysis of all patients yielded 10 additional patients with possible TB pathologic findings, bringing the total with TB-associated histology to 145 (63.8\%) patients. Additionally, there were $82(36.2 \%)$ cases with no evidence of TB, despite correct organ targeting (Table 1).

The reanalysis performed on the $\mathrm{CN}$ cases in the first interpretation confirmed all $\mathrm{CN}$ cases; $20(95.2 \%)$ of 21 necrosis cases that were determined to be non- $\mathrm{CN}$ in the first interpretation, such as simple necrosis, fibrinoid necrosis, coagulation necrosis, and abscess, were reinterpreted to be highly PCN cases (Table 2).

Additionally, of the findings under other in the first interpretation, 10 cases were reclassified as possible TB pathologic findings. Of these, three possible pulmonary TB cases were

TABLE 1

Patients' baseline characteristics

\begin{tabular}{lc}
\hline \multicolumn{1}{c}{ Category } & Number \\
\hline Patient number & 227 \\
Sex & \\
Male & 114 \\
Female & 113 \\
Age (years) & $59.4(18-91)$ \\
First histopathological interpretation & \\
$\quad$ (per interpretation sheets) & $80(35.2 \%)$ \\
CGI with CN & $49(21.6 \%)$ \\
CGI & $6(2.6 \%)$ \\
CN & $92(40.5 \%)$ \\
Other & \\
Second histopathological interpretation & $145(63.9 \%)$ \\
(after reanalysis of biopsy results) & $82(36.1 \%)$ \\
Possible TB pathologic findings & \\
Other diseases & \\
(correct targeting but no evidence of TB) & 181 \\
Site & 22 \\
Lung & 9 \\
Pleura & 5 \\
Bone (spine) & 2 \\
Neck lymph node & 2 \\
Colon & 6 \\
Pericardium & 2 \\
Genitourinary tract & 2 \\
Epididymis & 1 \\
Bladder & 1 \\
Endometrium & \\
Ureter & \\
\hline
\end{tabular}

TABLE 2

Results of the second interpretation relating to necrosis

\begin{tabular}{lcc}
\hline \multicolumn{1}{c}{ First interpretation } & Second interpretation & Case $N$ \\
\hline $\mathrm{CN}$ & $\mathrm{CN}$ & 86 of 86 \\
Necrosis & PCN & 12 of 13 \\
Fibrinoid necrosis & PCN & 2 of 2 \\
Coagulation necrosis & PCN & 4 of 4 \\
Abscess & PCN & 2 of 2 \\
\hline
\end{tabular}

initially interpreted as chronic inflammation with necrosis, coagulation necrosis, and infarction. The seven cases reclassified as possible extrapulmonary cases comprised two cases of tuberculous pleurisy, which had been initially interpreted as chronic inflammation with fibrinoid necrosis, one case of genitourinary TB corrected from necrotic tissue, and four cases of tuberculous lymphadenitis initially interpreted as simple necrosis $(N=3)$ and chronic inflammation with necrosis $(N=1)$.

Comparison of biopsy results according to $\mathbf{T B}$ sites. The initial biopsy interpretation results were that only $63(34.8 \%)$ of 181 patients with pulmonary TB were confirmed to have caseating granuloma, whereas $36(19.9 \%)$ and $6(3.3 \%)$ patients had only CGI or CN, respectively (Table 3). Among 46 patients with extrapulmonary TB, $16(34.8 \%)$ patients had only caseating granuloma, and $14(30.4 \%)$ patients had only CGI. The remaining 16 patients $(34.8 \%)$ had other findings.

The reanalysis resulted in $108(59.7 \%)$ cases of possible TB pathologic findings of 181 patients with pulmonary TB, whereas the remaining 73 patients $(40.3 \%)$ had other findings. Of 46 patients with extrapulmonary TB, 37 (80.4\%) patients showed possible TB pathologic findings, and 9 (19.6\%) patients had other findings, showing that, compared with pulmonary TB, extrapulmonary TB yielded possible TB pathologic findings more frequently $(P=0.010)$ (Table 3$)$.

Analysis of patients with other histological findings. The analyses of patients showing other histological findings yielded three types: normal tissue of the given organ; abnormal findings, such as chronic inflammation or fibrous tissue, that are non-specific to TB; and findings of clearly different diseases, such as bronchiolitis obliterans with organizing pneumonia, cancer, and pneumonia, where there was no suspicion of a TB diagnosis (which are two different coexisting diagnoses). Non-specific chronic inflammation was the predominant finding (Table 4).

Histopathological findings of lesion sites according to biopsy method. Of 181 patients with pulmonary TB, 140 (77.3\%) patients were subjected to transbronchial lung biopsy (TBLB) followed by percutaneous lung biopsy (PCLB) $(N=38 ; 21.0 \%)$ and open biopsy $(N=3 ; 1.7 \%)$. TBLB yielded 50 cases of caseating granuloma (35.7\%), 23 cases of CGI (16.4\%), and 2 cases of $\mathrm{CN}(1.4 \%)$, whereas PCLB yielded 12 cases of caseating granuloma (31.6\%), 13 cases of CGI $(34.2 \%)$, and 4 cases of $\mathrm{CN}(10.5 \%)$. These results showed that PCLB had a higher rate $(76.3 \%)$ of identifying any case of caseating granuloma, CGI, and CN than TBLB (53.6\%), a difference that was significant $(P=0.012)$ (Table 5). PCLB yielded three more cases of possible TB pathologic findings, showing that PCLB had a higher rate $(84.2 \%)$ of identifying possible TB pathologic findings than TBLB (53.6\%), a difference that was significant $(P=0.001)$.

Of nine osseous TB cases, open biopsy and needle biopsy were performed on six $(66.7 \%)$ and three $(33.3 \%)$ patients, 
TABLE 3

Histopathological findings according to lesion site (Pearson's $\chi^{2}$ test)

\begin{tabular}{|c|c|c|c|c|c|c|c|c|}
\hline \multirow[b]{2}{*}{ Lesion sites } & \multirow[b]{2}{*}{ Total } & \multicolumn{5}{|c|}{ First interpretation $N(\%)$} & \multicolumn{2}{|c|}{ Second interpretation $N(\%)$} \\
\hline & & Caseating granuloma & CGI & $\mathrm{CN}$ & One or both of CGI or CN & Others & PTBPF & Others \\
\hline Pulmonary TB & 181 & $63(34.8)$ & $36(19.9)$ & $6(3.3)$ & $105(58.0)$ & $76(42.0)$ & $108(59.7)$ & $73(40.3)$ \\
\hline \multicolumn{9}{|l|}{ ЕРТВ } \\
\hline Pleura & 22 & $5(22.7)$ & $8(36.4)$ & & $13(59.1)$ & $9(40.9)$ & $15(68.2)$ & $7(31.8)$ \\
\hline Bone & 9 & $5(55.6)$ & $3(33.3)$ & & $8(88.9)$ & $1(11.1)$ & $8(88.9)$ & $1(11.1)$ \\
\hline GU tract & 6 & $4(66.7)$ & $1(16.7)$ & & $5(83.3)$ & $1(16.7)$ & $6(100)$ & 0 \\
\hline Neck LN & 5 & $1(20.0)$ & & & $1(20.0)$ & $4(80.0)$ & $5(100)$ & 0 \\
\hline Pericardium & 2 & $1(50.0)$ & $1(50.0)$ & & $2(100)$ & & $2(100)$ & 0 \\
\hline Colon & 2 & & $1(50.0)$ & & $1(50.0)$ & $1(50.0)$ & $1(50.0)$ & $1(50.0)$ \\
\hline EPTB total & 46 & $16(34.8)$ & $14(30.4)$ & 0 & $30(65.2)$ & $16(34.8)$ & $37(80.4)$ & $9(19.6)$ \\
\hline Total & 227 & $79(34.8)$ & $50(22.0)$ & $6(2.6)$ & $135(59.5)$ & $92(40.5)$ & $145(63.8)$ & $82(36.2)$ \\
\hline$P$ value & & 0.998 & 0.123 & 0.211 & 0.374 & & 0.010 & \\
\hline
\end{tabular}

$\mathrm{EPTB}=$ extrapulmonary TB; $\mathrm{GU}=$ genitourinary $; \mathrm{LN}=$ lymph node; $\mathrm{PTBPF}=$ possible TB pathologic findings.

respectively. Open biopsy yielded five cases $(83.3 \%)$ of caseating granuloma and one case $(16.7 \%)$ of CGI, whereas needle biopsy yielded two cases $(66.7 \%)$ of CGI.

Additional histological findings indicative of possible TB. CGI with $\mathrm{CN}$ is a characteristic histopathological finding of TB (Figure 1). However, chronic inflammation, coagulation necrosis (infarction), fibrinoid necrosis, abscess, and/or hyalinized lesions found in TB-positive cases in this series can be indicative of possible TB, especially in the limited small biopsy samples (Figure 2).

\section{DISCUSSION}

In this study, we investigated the frequency of caseating granuloma, a histopathological feature characteristic of TB. Only one-third of the patients with clinically confirmed TB had caseating granuloma, and the combined rate of confirmed $\mathrm{CN}$ or CGI was relatively low in patients with pulmonary and extrapulmonary TB (58\% and 65.2\%, respectively). Although the reason for this relatively low histopathological confirmation rate of TB-suspected lesions in clinical patients is unclear, it may be attributable to the uncertainty of targeting the exact lesion site during biopsy collection. In cases of pulmonary TB, the exact lesion sites that would show typical pathologic findings for TB are commonly missed, despite the identification of infected lung tissue by histopathological examination.

In our reanalysis, a pathologist reanalyzed the initial biopsies with the knowledge that they were culture-confirmed TB cases; this reanalysis yielded a significantly higher confirmation rate of possible TB pathological findings in patients with extrapulmonary TB $(80.4 \% ; N=37 / 46)$ than in patients with pulmonary TB $(59.7 \% ; N=108 / 181 ; P=0.01)$. This finding leads us to assume that the targeting of extrapulmonary TB lesion sites was more effective than that of pulmonary TB lesion sites. Additionally, this result may be ascribed to the

TABLE 4

Other histopathological findings according to lesion site

\begin{tabular}{|c|c|c|c|c|c|c|}
\hline $\begin{array}{l}\text { Lesion } \\
\text { sites }\end{array}$ & $\begin{array}{c}\text { Chronic } \\
\text { inflammation }\end{array}$ & $\begin{array}{l}\text { Fibrous } \\
\text { tissue }\end{array}$ & $\begin{array}{c}\text { Non-significant } \\
\text { pathologic change }\end{array}$ & Cancer & Others & Total \\
\hline Lung & $48(65.8)$ & $3(4.2)$ & $2(2.8)$ & $15(20.8)$ & $5(6.9)$ & 73 \\
\hline Pleura & 7 (100) & & & & & 7 \\
\hline Bone & & & $1(100)$ & & & 1 \\
\hline Colon & $1(100)$ & & & & & 1 \\
\hline
\end{tabular}

Lung cancer includes squamous cell carcinoma (nine cases), small cell carcinoma (four cases), and adenocarcinoma (two cases). Others includes hamartoma, bullae, aspergillosis, emphysematous change, and edematous stroma. same targeting-related explanation for PCLB yielding a higher rate of identifying caseating granuloma or $\mathrm{CN}$ than TBLB.

Our targeting-related hypothesis is strongly supported by the difference in the sensitivities of the histopathological examinations depending on the method used to collect the biopsy specimen. For example, six of nine patients with osseous TB in our study underwent open biopsy, which confirmed five $(83.3 \%)$ cases of caseating granuloma and one case (16.7\%) of CGI.

The AFB stain test is simple and cost-effective. However, it has certain drawbacks: it requires 5,000-10,000 bacteria/mL for detection, and it is unable to distinguish between TB and non-TB Mycobacterium. Its sensitivity is $22-78 \%$ (relatively low), whereas its specificity is reportedly $99-100 \% .{ }^{8-11}$ Mycobacterial culture is more sensitive (80-85\%) than AFB staining, requiring $\geq 100$ bacteria/mL for detection, but the growth of TB bacilli requires 3-6 weeks or more. ${ }^{8-11}$ Polymerase chain reaction $(\mathrm{PCR})$ can detect mycobacteria directly from a specimen, requiring only a small number of bacteria for detection, and it is known to be an effective test method for early detection because of its high sensitivity and specificity and rapid test results. However, its high sensitivity poses the problem of detecting dead mycobacteria or a past infection. ${ }^{12,13}$

In cases of extrapulmonary $\mathrm{TB}$ in particular, the low number of mycobacteria in a TB lesion often leads to the failure to detect the TB bacilli; furthermore, collecting a biopsy specimen from an extrapulmonary TB lesion is often difficult. Therefore, compared with pulmonary TB, the microbiological detection method has a low success rate for extrapulmonary TB. ${ }^{14,15}$ In such cases, the histopathological confirmation of caseating granuloma can contribute greatly to the clinical diagnosis of TB.

TABLE 5

Results according to TB biopsy method (Pearson's $\chi^{2}$ test)

\begin{tabular}{lcccc}
\hline \multicolumn{1}{c}{ Biopsy findings } & TBLB $N(\%)$ & PCLB $N(\%)$ & Open biopsy $N(\%)$ & $P$ value \\
\hline Caseating granuloma & $50(35.7)$ & $12(31.6)$ & $1(33.3)$ & 0.635 \\
CGI & $23(16.4)$ & $13(34.2)$ & 0 & 0.016 \\
CN & $2(1.4)$ & $4(10.5)$ & 0 & 0.006 \\
Others & $65(46.4)$ & $9(23.7)$ & $2(66.7)$ & \\
\hline CGI or CN & $75(53.6)$ & $29(76.3)$ & $1(33.3)$ & 0.012 \\
Others & $65(46.4)$ & $9(23.7)$ & $2(66.7)$ & \\
\hline PTBPF & $75(53.6)$ & $32(84.2)$ & $1(33.3)$ & 0.001 \\
Others & $65(46.4)$ & $6(15.8)$ & $2(66.7)$ & \\
\hline Total $(N=181)$ & 140 & 38 & 3 & \\
\hline PTBPF $=$ possible TB pathologic findings. & & &
\end{tabular}




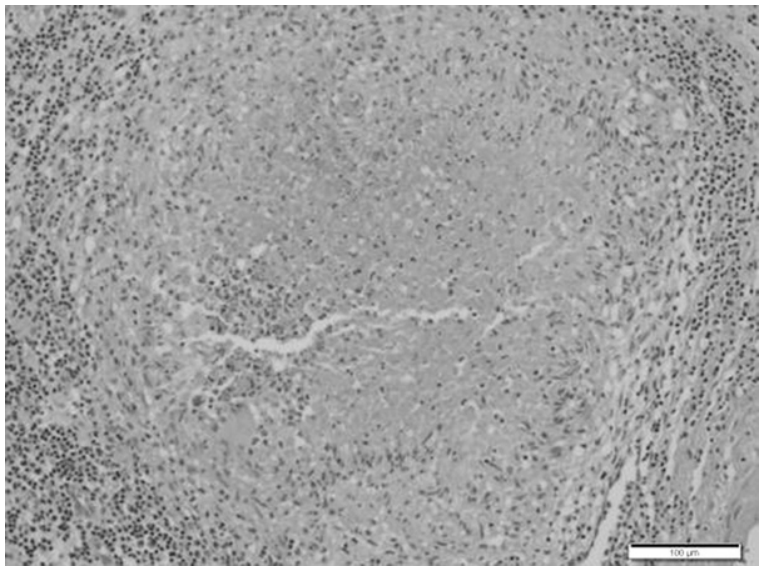

FIGURE 1. Chronic granulomatous inflammation with central CN (soft tubercle). A rim of epithelioid cells and lymphocytes around the central necrosis is present. Hematoxylin and eosin stain. Scale bar $=100 \mu \mathrm{m}$.

A study by Merino and others ${ }^{16}$ revealed a positive AFB stain test rate of $45.2 \%$ in patients with confirmed spinal TB, thus showing the diagnostic challenge of microbiological detection methods.

CGI is divided into soft and hard tubercles depending on the presence (soft) or absence (hard) of central $\mathrm{CN}$ in the tubercle formation of granulomatous lesions with epithelioid cells and lymphocytes. A TB diagnosis is straightforward in cases of soft and hard tubercles that are entirely harvested during biopsy. However, if only a part of a hard tubercle is harvested, CGI may be misinterpreted as simple chronic inflammation. $\mathrm{CN}$ is a specialized type of liquefaction necrosis that is composed of coagulation and liquefaction necrosis patterns. If a limited specimen size is obtained through endoscopy or a percutaneous needle biopsy instead of a sufficient volume being secured through surgical excision, the pathological state will be interpreted on the basis of partial $\mathrm{CN}$ components. Consequently, possible TB pathologic findings may arise from an ambiguous diagnosis because of the pathologist's subjective judgment, such as vague $\mathrm{CN}$, or the initial interpretation of a specimen that shows only partial components of $\mathrm{CN}$ as being a non-CN condition, such as coagulation necrosis, fibrinoid necrosis, and abscesses that resemble $\mathrm{CN}$.

If a limited sample yields a result of non-TB-specific caseating granuloma, a pathologist faces difficulties in diagnosing TB. Nevertheless, given the high number of TB cases confirmed in retrospective studies such as our study from cases initially interpreted as infarction, necrosis, fibrinoid necrosis, and coagulation necrosis, performing an additional culture test or PCR test to obtain definite confirmation is crucial when TB-nonspecific but suspected histopathological findings present as a result of the biopsy from clinically
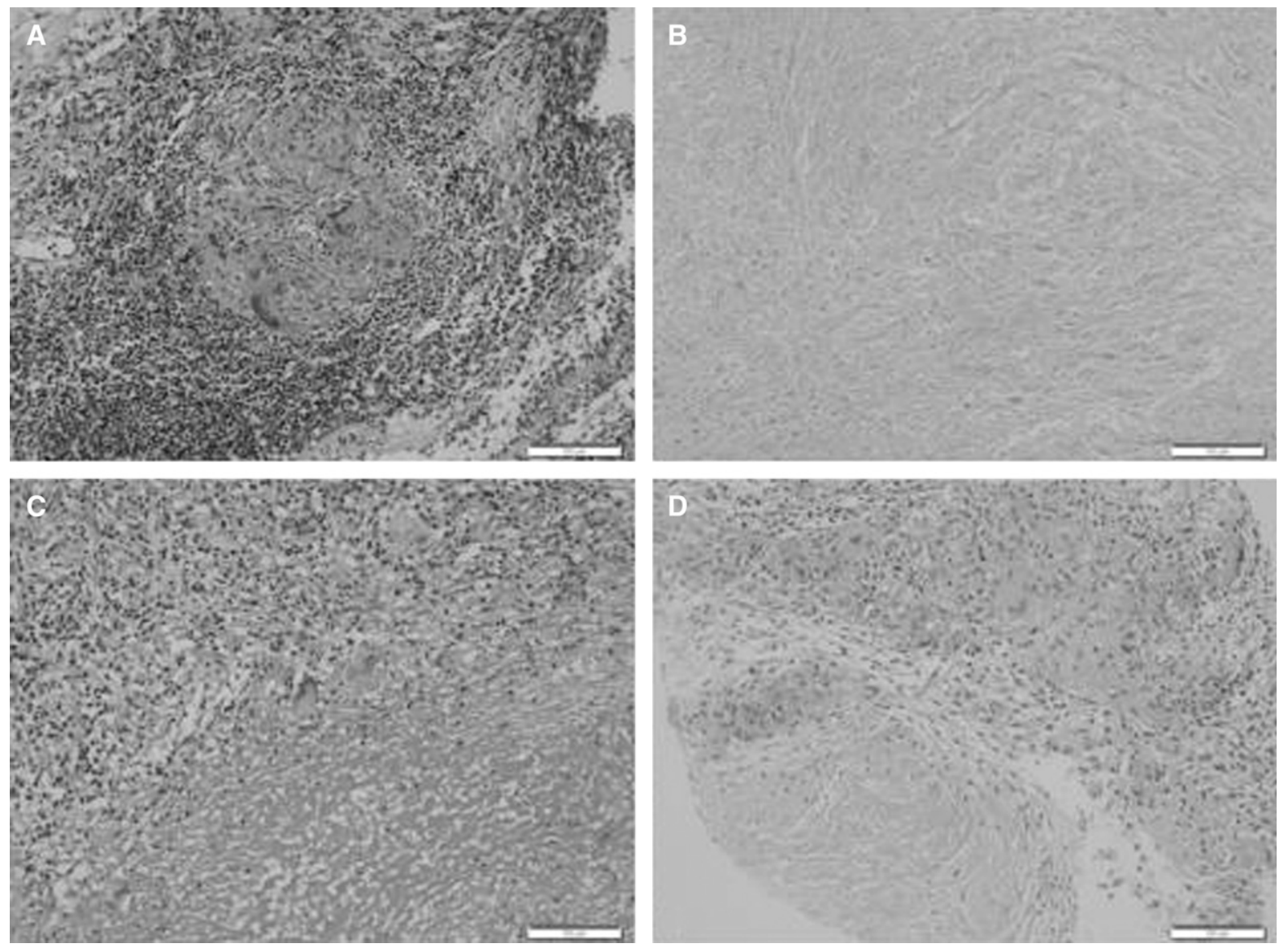

FIGURE 2. (A) Chronic granulomatous inflammation without central CN (hard tubercle). A collection of Langhans giant and epithelioid cells is visible. (B) Coagulation necrosis composed of devitalized cells and stromal frameworks. (C) Chronic granulomatous inflammation around the fibrinoid necrosis (lower). (D) Chronic granulomatous inflammation around the hyaline change (lower left). Hematoxylin and eosin stain. Scale bar $=100 \mu \mathrm{m}$. 
suspected TB cases. In TB lymphadenitis, in particular, all four cases interpreted in the initial reading as other types of necrosis (three necrotic tissue cases and one chronic inflammation with necrosis case) were interpreted as TB-suspected findings in the reanalysis. Thus, we verified that, in the lymph node histologic examination of simple necrosis cases, the presence of TB bacilli cannot be excluded. In extrapulmonary TB, CGI with some necrosis is likely actually a feature of caseating granuloma. In such cases, the possibility of $\mathrm{TB}$ should be considered in regions with high TB prevalence.

In our study, TB concomitant with lung cancer was verified in $15(8.3 \%)$ of 181 patients diagnosed with pulmonary TB; the cases were squamous cell carcinoma (nine), adenocarcinoma (two), and small cell carcinoma (four). Studies have shown that lung disease is an independent risk factor for lung cancer, with pulmonary TB showing a 2.1-fold risk, presumably because of DNA damage caused by reactive oxygen species (ROS) induced by the structural components of the TB bacterial cell wall, increased mutations caused by chronic inflammation, and diminished immune function caused by the cancer and its treatment. ${ }^{17,18}$ Therefore, we confirmed that, even if a histological examination yields a finding of chronic inflammation, the possibility of a TB diagnosis should not be excluded given that histologically confirmed lung cancer was relatively frequently concurrent with pulmonary $\mathrm{TB}$ and that the histological finding of chronic inflammation accounted for $24.2 \%$ of the total histological findings. In particular, $13(59.1 \%)$ of 22 pleural TB cases showed either CGI or CN, whereas the remaining 9 cases yielded chronic inflammation findings only. This result implies that even findings of only chronic inflammation should not eliminate TB.

In conclusion, the analyses of the histologic findings of patients with culture-confirmed TB yielded only one-third concordance rates in both pulmonary and extrapulmonary TB cases with respect to the confirmation of caseating granulomas, and the rates of confirming either CN or CGI were $58 \%$ and $65.2 \%$ for pulmonary and extrapulmonary TB cases, respectively. Therefore, in regions with high TB prevalence, TB should not be eliminated, although the patient does not present with CGI or CN. In particular, even in patients in whom only necrosis is identified, the presence of $\mathrm{CN}$ is possible; thus, it is important for clinicians to consider that cases reported as other types of necrosis may also be $\mathrm{CN}$.

Received October 19, 2014. Accepted for publication November 11, 2014.

Published online December 8, 2014.

Financial support: This work was supported by grants from the Clinical Medicine Research Institute at Chosun University Hospital (2010).

Authors' addresses: Kyung Min Bae, Hyung Ho Kim, Woo Jin Lee, $\mathrm{Na}$ Ra Yun, and Dong-Min Kim, Department of Internal Medicine, Chosun University College of Medicine, Gwangju, South Korea, E-mails: bkm8023@naver.com, gudgh0318@naver.com, blu2mania@ gmail.com, shine@chosun.ac.kr, and drongkim@chosun.ac.kr.Sung-Chul Lim, Department of Pathology, Chosun University College of Medi- cine, Gwangju, South Korea, E-mail: sclim@chosun.ac.kr. Choon-Mee Kim, Division of Natural Medical Sciences, College of Health Science, Chosun University, Gwangju, South Korea, E-mail: choonmee@ chosun.ac.kr.

\section{REFERENCES}

1. Lönnroth K, Raviglione M, 2008. Global epidemiology of tuberculosis: prospects for control. Semin Respir Crit Care Med 29: 481-491.

2. Dye C, Watt CJ, Bleed DM, Hosseini SM, Raviglione MC, 2005. Evolution of tuberculosis control and prospects for reducing tuberculosis incidence, prevalence, and deaths globally. JAMA 293: 2767-2775.

3. Klatt EC, 2013. Pathology of Tuberculosis. The Internet Pathology Laboratory for Medical Education. Available at: http:// www-medlib.med.utah.edu/WebPath/TUTORIAL/MTB/MTB .html. Accessed October 10, 2013.

4. Ajantha GS, Shetty PC, Kulkarni RD, Biradar U, 2013. PCR as a diagnostic tool for extra-pulmonary tuberculosis. J Clin Diagn Res 7: 1012-1015.

5. Chakravorty S, Sen MK, Tyagi JS, 2005. Diagnosis of extrapulmonary tuberculosis by smear, culture, and using universal sample processing technology. J Clin Microbiol 43: 4357-4362.

6. Cutler RR, Baithun SI, Doran HM, Wilson P, 1994. Association between the histological diagnosis of tuberculosis and microbiological findings. Tuber Lung Dis 75: 75-79.

7. Park DY, Kim JY, Choi KU, Lee JS, Lee CH, Sol MY, Suh KS, 2003. Comparison of polymerase chain reaction with histopathologic features for diagnosis of tuberculosis in formalin-fixed, paraffin-embedded histologic specimens. Arch Pathol Lab Med 127: 326-330.

8. Kivihya-Ndugga L, van Cleeff M, Juma E, Kimwomi J, Githui W, Oskam L, Schuitema A, van Soolingen D, Nganga L, Kibuga D, Odhiambo J, Klatser P, 2004. Comparison of PCR with the routine procedure for diagnosis of Tuberculosis in a population with high prevalences of tuberculosis and human immunodeficiency virus. J Clin Microbiol 42: 1012-1015.

9. Almeda J, Garcia A, Gonzalez J, Quinto L, Ventura PJ, Vidal R, Rufi G, Martinez JA, Jimenez de Anta MT, Trilla A, Alonso PL, 2000. Clinical evaluation of an in-house IS6110 polymerase chain reaction for diagnosis of tuberculosis. Eur J Clin Microbiol Infect Dis 19: 859-867.

10. American Thoracic Society, 2000. Diagnostic standards and classification of tuberculosis in adults and children. Am J Respir Crit Care Med 161: 1376-1395.

11. Kim JH, Jang SJ, Moon DS, Park YJ, 2003. Evaluation of two PCR-hybridization methods for the detection of Mycobacterium tuberculosis. Korean J Lab Med 23: 32-38.

12. Lee KE, Cho JH, Moon YH, 1998. Comparison of stain methods with PCR and culture for the detection of Mycobacterium tuberculosis in the sputum. Korean J Clin Pathol 18: 201-207.

13. Shin WS, 1992. Diagnosis of tuberculosis; serodiagnosis and molecular biologic approach. Tuberc Respir Dis (Seoul) 39: 1-6.

14. Long MD, Ellis E, 2007. Canadian Tuberculosis Standards, 6th ed. Ottawa, Canada: Public Health Agency.

15. Golden MP, Vikram HR, 2005. Extrapulmonary tuberculosis: an overview. Am Fam Physician 72: 1761-1768.

16. Merino P, Candel FJ, Gestoso I, Baos E, Picazo J, 2012. Microbiological diagnosis of spinal tuberculosis. Int Orthop 36: 233-238.

17. Brenner AV, Wang Z, Kleinerman RA, Wang L, Zhang S, Metayer C, Chen K, Lei S, Cui H, Lubin JH, 2001. Previous pulmonary diseases and risk of lung cancer in Gansu Province, China. Int J Epidemiol 30: 118-124.

18. Falagas ME, Kouranos VD, Athanassa Z, Kopterides P, 2010. Tuberculosis and malignancy. Q J Med 103: 461-487. 